Tôhoku Math. Journ.

23(1971), 1-11.

\title{
ON CONFORMALLY FLAT SPACES SATISFYING A CERTAIN CONDITION ON THE RICCI TENSOR
}

\author{
Kouei SeKigawa AND Hitoshi TAKagi
}

(Received February 2, 1970)

1. Introduction. The Riemannian curvature tensor $R$ of a locally symmetric Riemannian manifold $(M, g)$ satisfies

$$
R(X, Y) \cdot R=0 \quad \text { for any tangent vectors } X \text { and } Y \text {, }
$$

where the endomorphism $R(X, Y)$ operates on $R$ as a derivation of the tensor algebra at each point of $M$.

Let $R_{1}$ be the Ricci tensor of $(M, g)$. Then $\left(^{*}\right)$ implies in particular

$$
R(X, Y) \cdot R_{1}=0 \quad \text { for any tangent vectors } X \text { and } Y .
$$

In the present paper we shall prove

THEOREM A. Let $M^{m}(m \geqq 3)$ be an m-dimensional connected complete conformally flat space satisfying the condition (**). Then $M^{m}$ is one of the following manifolds:

( I) A space of constant curvature.

(II) A locally product space of a space of constant curvature $K(\neq 0)$ and a space of constant curvature $-K$.

(III) A locally product space of a space of constant curvature $K(\neq 0)$ and a 1-dimensional space.

The authors wish to express their sincere thanks to Prof. S. Tanno who gave them many valuable suggestions and guidances, and also thanks to Prof. S. Sawaki who gave the authors many guidances.

2. Conformally flat cases of dimension $\boldsymbol{m}>3$. Let $M^{m}(m>3)$ be a connected conformally flat spaces, then the curvature tensor $R$ of $M^{m}$ is given by 


$$
R(X, Y)=(1 /(m-2))(A X \wedge Y+X \wedge A Y)-(\operatorname{trace} A /(m-1)(m-2)) X \wedge Y,
$$

for any tangent vectors $X$ and $Y$ of $M^{m}$, where $A$ denotes a field of symmetric endomorphism which corresponds to the Ricci tensor $R_{1}$, that is, $R_{1}(X, Y)=g(A X, Y)$, and $X \wedge Y$ denotes the endomorphism which maps $Z$ upon $g(Y, Z) X-g(X, Z) Y$.

At a point of $M^{m}$, let $\left\{e_{1}, e_{2}, \cdots, e_{m}\right\}$ be an orthonormal basis of the tangent space such that $A e_{i}=\lambda_{i} e_{i}, 1 \leqq i \leqq m$. Then the equation (2.1) implies

$$
R\left(e_{i}, e_{j}\right)=((m-1)(m-2))^{-1}\left((m-1)\left(\lambda_{i}+\lambda_{j}\right)-\sum_{k=1}^{m} \lambda_{k}\right) e_{i} \wedge e_{j}
$$

Now by the equation $(* *)$ and

$$
\left[R\left(e_{i}, e_{j}\right) \cdot R_{1}\right]\left(e_{k}, e_{h}\right)=-R_{1}\left(R\left(e_{i}, e_{j}\right) e_{k}, e_{h}\right)-R_{1}\left(e_{k}, R\left(e_{i}, e_{j}\right) e_{h}\right),
$$

we have

$$
\left(\lambda_{i}-\lambda_{j}\right)\left((m-1)\left(\lambda_{i}+\lambda_{j}\right)-\sum_{k=1}^{m} \lambda_{k}\right)=0, \text { for } i \neq j .
$$

In this paper, the indices $i, j, k, h, \cdots$ run from 1 to $m$.

LEMMA 2.1. At each point of $M^{m}$, the rank of $R_{1}$ is $m, m-1$, or 0 .

PROOF. If there exists an integer $r(1<r<m)$ such that $\lambda_{1}=\cdots=\lambda_{r}=0$, $\lambda_{r+1} \neq 0, \cdots, \lambda_{m} \neq 0$, and if we put $\Lambda=\sum_{k=1}^{m} \lambda_{k}$, then (2.2) implies

$$
\begin{gathered}
(m-1) \lambda_{r+1}-\Lambda=0, \\
\cdots \\
(m-1) \lambda_{m}-\Lambda=0 .
\end{gathered}
$$

Hence $\lambda_{r+1}=\cdots=\lambda_{m}=\lambda \neq 0$. Again (2.2) implies $(m-1) \lambda-(m-r) \lambda=(r-1) \lambda=0$, that is, $\lambda=0$ which is a contradiction.

Q. E. D.

LEMMA 2.2. If all the $\lambda_{i}$ 's have the same sign at a point of $M^{m}$, then $\lambda_{1}=\lambda_{2}=\cdots \lambda_{m}=\lambda$, at the point.

PROOF. If there exists an integer $r(1 \leqq r<m)$ such that $\lambda_{1}=\cdots=\lambda_{r}=\lambda$, $\lambda_{r+1} \neq \lambda, \cdots, \lambda_{m} \neq \lambda$, then (2.2) implies 


$$
\begin{gathered}
(m-1)\left(\lambda+\lambda_{r+1}\right)-\Lambda=0, \\
\cdots \cdots \\
(m-1)\left(\lambda+\lambda_{m}\right)-\Lambda=0 .
\end{gathered}
$$

Heace $\lambda_{r+1}=\cdots=\lambda_{m}=\mu \neq 0$. Again (2.2) implies $(m-1)(\lambda+\mu)-r \lambda-(m-r) \mu=0$, that is,

$$
(m-r-1) \lambda=(1-r) \mu .
$$

Then, as $m>3$, from (2.3) we have $r \neq 1, m-1$. But from (2.3) we have also $\lambda \mu<0$. This is a contradiction.

Q.E.D.

Now we have

Proposition 2.3. Let $M^{m}(m>3)$ be a connected conformally flat space satisfying the condition (**). If the Ricci form $R_{1}$ is definite at least at one point of $M^{m}$, then $M^{m}$ is a space of constant curvature.

PROOF. If the Ricci form $R_{1}$ is positive (resp. negative) definite at some point $x_{0} \in M^{m}$, then, by the continuity argument for the characteristic polynomial of $A$, $R_{1}$ is positive (resp. negative) definite near $x_{0}$ in $M^{m}$. Thus, let $W=\left\{x \in M^{m} ; R_{1}\right.$ is positive (resp. negative) definite at $x\}$, which is an open set. Let $W_{0}$ be a connected component of $x_{0}$ in $W$. Then by lemma $2.2, \lambda_{1}=\cdots=\lambda_{m}=\lambda$, on $W_{0}$ and $\lambda(x)$ is a differentiable function on $W_{0}$, since $\lambda(x)=$ trace $A / m$. Now, the open submanifold $W_{0}$ becomes a conformally flat space by the Riemannian metric which is the restriction of $g$ to $W_{0}$. Thus $W_{0}$ becomes an Einstein space by the induced metric from $M^{m}$. As $m>3, \lambda(x)$ is a constant function on $W_{0}$. Hence, (2.1) implies that $W_{0}$ is a space of constant curvature $\lambda /(m-1)$. Therefore, by the connectivity of $M^{m}$ and the continuity argument for the characteristic polynomial of $A$, it follows that $W_{0}=M^{m}$.

Q.E.D.

Next, we assume that the Ricci form $R_{1}$ is non-degenerate and indefinite at some point $x_{0} \in M^{m}$. Then, from the proof of lemma 2.2, there exists an integer $r(1<r<m-1)$ such that $\lambda_{1}=\cdots=\lambda_{r}=\lambda>0$, and $\lambda_{r+1}=\cdots=\lambda_{m}=\mu<0$, at $x_{0}$. By the continuity argument for the characteristic polynomial of $A$, let $W=\left\{x \in M^{m}\right.$; $R$ is non-degenerate and indefinite at $x\}$, which is an open set. Let $W_{0}$ be a connected component of $x_{0}$ in $W$.

Then it follows that $r$ is constant on $W_{0}$ and non-zero eigenvalues, $\lambda(x)>0$, and $\mu(x)<0$ are differentiable functions on $W_{0}$, since, if $m \neq 2 r$, then $\lambda(x)=F(x)$, $\mu(x)=G(x)$, or $\lambda(x)=G(x), \mu(x)=F(x) x \in W_{0}$, where 


$$
\begin{gathered}
F(x)=((1-r) /(m-1)(m-2 r)) \text { trace } A, \\
G(x)=((m-r-1) /(m-1)(m-2 r)) \text { trace } A,
\end{gathered}
$$

and if $m=2 r$, then $\lambda(x)={ }^{2 r} \sqrt{(-1)^{r} \operatorname{det} A}, \mu(x)=-{ }^{2 r} \sqrt{(-1)^{r} \operatorname{det} A}, x \in W_{0}$.

We define two distributions on $W_{0}$ as follows:

$$
\begin{aligned}
& T_{1}(x)=\left\{X \in M_{x}^{m} ; A X=\lambda(x) X\right\}, \\
& T_{2}(x)=\left\{X \in M_{x}^{m} ; A X=\mu(x) X\right\} .
\end{aligned}
$$

LEMMA 2.4. $T_{1}(x)$ and $T_{2}(x)$ are differentiable on $W_{0}$.

Proof is given by the slight modifications of the arguments in [3].

By lemma 2.4, for any $x \in W_{0}$ we may choose a differentiable field of orthonormal basis $\left\{X_{1}, X_{2}, \cdots, X_{m}\right\}$ near $x$ in $W_{0}$ in such a way that $\left\{X_{1}, \cdots, X_{r}\right\}$ and $\left\{X_{r+1}, \cdots, X_{m}\right\}$ are bases near $x$ in $W_{0}$ for $T_{1}$ and $T_{2}$, respectively.

By making use of (2.1) and (2.3), we have

LEMMA 2.5. With respect to the basis $\left\{X_{1}, X_{2}, \cdots, X_{m}\right\}$, we have

$$
\begin{gathered}
R\left(X_{a}, X_{b}\right)=K X_{a} \wedge X_{b}, \\
R\left(X_{u}, X_{v}\right)=-K X_{u} \wedge X_{v},
\end{gathered}
$$

and otherwise zero, where $K=(\lambda-\mu) /(m-2)$ and $1 \leqq a, b, c, \cdots \leqq r, r+1$ $\leqq u, v, w, \cdots \leqq m$.

Now, in general, for a differentiable local field of orthonormal basis $\left\{X_{1}, X_{2}\right.$, $\left.\cdots, X_{m}\right\}$ in a Riemannian manifold $(M, g)$, we may put

$$
\nabla_{\mathbf{x}_{i}} X_{j}=\sum_{k=1}^{m} \gamma_{i j k} X_{k}
$$

where $\nabla_{\mathbf{x}}$ denotes covariant differentiation for the Riemannian connection constructed by $g$, and $\gamma_{i j k}=-\gamma_{i k j}$.

PROPOSITION 2.6. Let $M^{m}(m>3)$ be a connected conformally flat space satisfying the condition (**). If the Ricci form $R_{1}$ is non-degenerate and indefinite of signature $2 r-m$ at least at one point of $M^{m}$, then $M^{m}$ is a locally product space of an r-dimensional space of constant curvature $K$ and an $(m-r)$-dimensional space of constant curvature $-K$, where $1<r<m-1$. 
PROOF. Taking account of (2.4) and (2.5), we have

$$
\begin{aligned}
\left(\nabla_{\mathbf{x}_{u}} R\right)\left(X_{a}, X_{b}\right)= & X_{u} K X_{a} \wedge X_{b}+K \sum_{j=1}^{m} \gamma_{u a i} X_{i} \wedge X_{b} \\
& +K \sum_{i=1}^{m} \gamma_{u b i} X_{a} \wedge Y_{i}-K \sum_{c=1}^{r} \gamma_{u a c} X_{c} \wedge X_{b} \\
& -K \sum_{c=1}^{r} \gamma_{u b c} X_{a} \wedge X_{c} \\
= & X_{u} K X_{a} \wedge X_{b}+K \sum_{v=r+1}^{m} \gamma_{u a v} X_{v} \wedge X_{b}+K \sum_{v=r+1}^{m} \gamma_{u b v} X_{a} \wedge X_{v}, \\
\left(\nabla_{\mathbf{x}_{a}} R\right)\left(X_{b}, X_{u}\right)= & K \sum_{v=r+1}^{m} \gamma_{a b v} X_{v} \wedge X_{u}-K \sum_{c=1}^{r} \gamma_{a u c} X_{b} \wedge X_{c}, \\
\left(\nabla_{\mathbf{x}_{b}} R\right)\left(X_{u}, X_{a}\right)= & -K \sum_{c=1}^{r} \gamma_{b u c} X_{c} \wedge X_{a}+K \sum_{v=r+1}^{m} \gamma_{b a v} X_{u} \wedge X_{v} .
\end{aligned}
$$

By the second Bianchi identity, we have $X_{u} K=0$, and $\gamma_{u v a}=0$. Similarly we have $X_{a} K=0$, and $\gamma_{a b u}=0$. Where $a, b=1, \cdots, r ; u, v=r+1, \cdots, m$.

Thus $W_{0}$ is a locally product space of an $r$-dimensional space of constant curvature $K$ and an $(m-r)$-dimensional space of constant curvature $-K$. Therefore, by the connectivity of $M^{m}$ and the continuity argument for the characteristic polynomial of $A$, it follows that $W_{0}=M^{m}$. Q. E. D.

Lastly, we assume that the rank of the Ricci form $R_{1}$ is $m-1$ at some point $x_{0} \in M^{m}$, and furthermore $M^{m}$ is complete. Then, from the proofs of lemma 2.1 and lemma 2.2 , and the continuity argument for the characteristic polynomial of $A$, the rank of $R_{1}$ is $m-1$ near $x_{0}$ in $M^{m}$. Thus let $W=\left\{x \in M^{m}\right.$; the rank of $R_{1}$ is $m-1$ at $\left.x\right\}$, which is an open set. Let $W_{0}$ be a connected component of $x_{0}$ in $W$. From the proof of lemma 2.1, we see that all the non-zero eigenvalues of $A$ at each point of $W_{0}$ are equal to each other, say, $\lambda$, and the non-zero eigenvalue $\lambda(x)$ is a differentiable function on $W_{0}$, since $\lambda(x)=$ trace $A /(m-1)$.

We define two distributions on $W_{0}$ as follows:

$$
\begin{aligned}
& T_{1}(x)=\left\{X \in M_{x}^{m} ; A X=\lambda(x) X\right\}, \\
& T_{0}(x)=\left\{X \in M_{x}^{m} ; A X=0\right\}, \quad x \in W_{0} .
\end{aligned}
$$

Corresponding to lemma 2.4, we have 
LEMMA 2.7. $T_{1}(x)$ and $T_{0}(x)$ are differentiable on $W_{0}$.

Thus, for any $x \in W_{0}$, we may choose a differentiable field of orthonormal basis $\left\{X_{1}, X_{2}, \cdots, X_{m}\right\}$ near $x$ in $W_{0}$ in such a way that $\left\{X_{1}, \cdots, X_{m-1}\right\}$ and $\left\{X_{m}\right\}$ are bases near $x$ in $W_{0}$ for $T_{1}$ and $T_{0}$, respectively. Corresponding to lemma 2.5 , we have

LEMMA 2.8. With respect to the basis $\left\{X_{1}, X_{2}, \cdots, X_{m}\right\}$, we have

$$
R\left(X_{a}, X_{b}\right)=K X_{a} \wedge X_{b}
$$

and otherwise zero, where $K=\lambda /(m-2)$, and $1 \leqq a, b, c, \cdots \leqq m-1$.

LEMMA 2.9. $T_{1}$ is involutive.

PROOF. Taking account of (2.5) and (2.6), we have

$$
\begin{aligned}
& \left(\nabla_{\mathbf{x}_{c}} R\right)\left(X_{a}, X_{b}\right)=X_{c} K X_{a} \wedge X_{b}+K \gamma_{c a m} X_{m} \wedge X_{b}+K \gamma_{c b m} X_{a} \wedge X_{m} \\
& \left(\nabla_{\mathbf{x}_{a}} R\right)\left(X_{b}, X_{c}\right)=X_{a} K X_{b} \wedge X_{c}+K \gamma_{a b m} X_{m} \wedge X_{c}+K \gamma_{a c m} X_{b} \wedge X_{m} \\
& \left(\nabla_{x_{b}} R\right)\left(X_{c}, X_{a}\right)=X_{b} K X_{c} \wedge X_{a}+K \gamma_{b c m} X_{m} \wedge X_{a}+K \gamma_{b a m} X_{c} \wedge X_{m}
\end{aligned}
$$

By the second Bianchi identity, we have

$$
\begin{aligned}
& \quad X_{c} K=0, \quad c=1, \cdots, m-1 \\
& \gamma_{a b m}-\gamma_{b a m}=0, \quad \text { for } \quad a \neq b, \quad a, b=1, \cdots, m-1 .
\end{aligned}
$$

By (2.8), $T_{1}$ is involutive.

Q.E. D.

For each $x \in W_{0}$, we denote by $M_{1}(x)$ the maximal integral manifold through $x$ of $T_{1}$. Then, by (2.7), $K$ is constant on each $M_{1}(x)$.

LEMMA 2.10. Each trajectory of $X_{m}$ is a geodesic.

PROOF. From (2.5) and lemma 2.8, we have

$$
\begin{aligned}
& \left(\nabla_{\mathbf{x}_{m}} R\right)\left(X_{a}, X_{b}\right)=X_{m} K X_{a} \wedge X_{b}+K \gamma_{m a m} X_{m} \wedge X_{b}+K \gamma_{m b m} X_{a} \wedge X_{m} \\
& \left(\nabla_{X_{a}} R\right)\left(X_{b}, X_{m}\right)=-K \sum_{c=1}^{m-1} \gamma_{a m c} X_{b} \wedge X_{c} \\
& \left(\nabla_{X_{b}} R\right)\left(X_{m}, X_{a}\right)=-K \sum_{c=1}^{m-1} \gamma_{b m c} X_{c} \wedge X_{a}
\end{aligned}
$$


By the second Bianchi identity, we have

$$
\begin{aligned}
(2.9) & \gamma_{m m a}=0, \\
(2.10) & \gamma_{a m b}=0, \\
\text { (2.11) } \quad X_{m} K+K\left(\gamma_{a m a}+\gamma_{b m b}\right)= & 0, \text { for } a \neq b, \quad a, b=1, \cdots, m-1 .
\end{aligned}
$$

Thus, from (2.9), it follows that $\nabla_{\mathbf{x}_{m}} X_{m}=0$.

Q. E. D.

From (2.11) we have

$$
\gamma_{1 m 1}=\gamma_{2 m 2}=\cdots=\gamma_{m-1 m m-1}
$$

Thus, from lemma 2.8, taking account of (2.9), (2.10) and (2.12), we have

$$
\begin{aligned}
R\left(X_{a}, X_{m}\right) X_{m}= & \nabla_{\mathbf{x}_{a}} \nabla_{\mathbf{x}_{m}} X_{m}-\nabla_{\mathbf{x}_{m}} \nabla_{\mathbf{x}_{a}} X_{m}-\nabla_{\left[\mathbf{x}_{a}, \mathbf{x}_{m}\right]} X_{m} \\
= & -X_{m} \gamma_{a m a} X_{a}-\sum_{c=1}^{m-1} \gamma_{a m a} \gamma_{m a c} X_{c} \\
& -\sum_{c=1}^{m-1} \gamma_{a m a} \gamma_{a m c} X_{c}+\sum_{c=1}^{m-1} \gamma_{m a c} \gamma_{c m c} X_{c} \\
= & -X_{m} \gamma_{a m a} X_{a}-\left(\gamma_{a m a}\right)^{2} X_{a}=0
\end{aligned}
$$

that is

$$
X_{m} \gamma_{a m a}+\left(\gamma_{a m a}\right)^{2}=0, \quad \text { for } \quad a=1, \cdots, m-1
$$

LEMMA 2.11. Any geodesic whose tangent belongs to $T_{0}$ at each $x \in W_{0}$ is infinitely extendible in $W_{0}$.

ProOF. For any $x \in W_{0}$, let $L(s)$ be a geodesic with arc length $s$, whose initial point is $x$ and initial direction at $x$ belongs to $T_{0}$. Then, by lemma 2.10 , for sufficiently small $s$ each tangent vector at $s$ of $L(s)$ belongs to $T_{0}$.

Thus, from (2.11) and (2.12), we have

$$
\frac{d^{2} K}{d s^{2}}+2 \frac{d K}{d s} \gamma_{a m a}+2 K \frac{d}{d s} \gamma_{a m a}=0
$$

that is, 


$$
2 K \frac{d^{2} K}{d s^{2}}-3\left(\frac{d K}{d s}\right)^{2}=0
$$

If $K>0$, then (2.14) implies

$$
\frac{d^{2}}{d s^{2}}(1 / \sqrt{K})=0
$$

If $K<0$, then (2.14) implies

$$
\frac{d^{2}}{d s^{2}}(1 / \sqrt{-K})=0
$$

Therefore, from (2.15) and (2.16), we have

$$
K=1 /(a s+b)^{2}, \text { and }-1 /(a s+b)^{2}, \text { respectively, }
$$

where $a$ and $b$ are certain constants. As a geodesic in $M^{m}, L(s)$ is infinitely extendible. If this geodesic does not lie in $W_{0}$, let $s_{0}$ be a point such that $L(s) \in W_{0}$ for $s<s_{0}$ but $L(s) \notin W_{0}$. The characteristic polynomial of $A$ at $L(s)$, $s<s_{0}$, is $(t-\lambda(s))^{m-1} t$. That of $A$ at $L\left(s_{0}\right)$ is therefore the limit as $s \rightarrow s_{0}$, namely, $\left(t-\lambda\left(s_{0}\right)\right)^{m-1} t$. But $\lambda\left(s_{0}\right)=\lim _{s \rightarrow s_{o}} \lambda(s)=\lim _{s \rightarrow s_{0}} \pm(m-2) /(a s+b)^{2}= \pm(m-2) /\left(a s_{0}+b\right)^{2}$ can not be 0 . This is a contradiction. It follows that $L\left(s_{0}\right) \in W_{0}$ Q. E. D.

PROPOSITION 2.12. Let $M^{m}(m>3)$ be a connected complete conformally flat space satisfying the condition (**). If the rank of the Ricci form $R$ is $m-1$ at least at one point of $M^{m}$, then $M^{m}$ is a locally product space of an $(m-1)$-dimensional space of constant curvature $K$ and a 1-dimensional space.

PROOF. From lemma 2.11, $K(s)$ must to be defined for any $s$ along $L(s)$. But, if $a \neq 0$ in (2.17), then $1 / \lambda$ will be 0 for $s=-b / a$ which is a contradiction. We have thus shown that $K$ is equal to a constant on each $L(s)$. Therefore, $K$ is constant on $W_{0}$. Then, from (2.11) and (2.12), we have $\gamma_{a m a}=0$, for $a=1$, $\cdots, m-1$. Thus, from (2.9) and (2.10), $T_{1}$ and $T_{0}$ are parallel. Therefore, $M^{m}$ is a locally product space of an $(m-1)$-dimensional space of constant curvature $K$ and a 1 -dimensional space.

Q.E. D.

3. 3-dimensional cases. Let $M$ be a 3-dimensional connected Riemannian manifold with the metric tensor $g$. Then the curvature tensor $R$ of $M$ is given by

$$
R(X, Y)=A X \wedge Y+X \wedge A Y-(\text { trace } A / 2) X \wedge Y
$$


for any tangent vectors $X$ and $Y$ of $M$, where $A$ is a symmetric endomorphism satisfying $R_{1}(X, Y)=g(A X, Y)$. Then (3.1) is obtained by putting $m=3$ in (2.1). This suggests that we may apply the similar ones as the arguments in $\S 2$ in this section.

At a point of $M$, let $\left\{e_{1}, e_{2}, e_{3}\right\}$ be an orthonormal basis of the tangent space such that $A e_{i}=\lambda_{i} e_{i}, i=1,2,3$. Then the condition $\left(^{* *}\right)$ is equivalent to

$$
\left(\lambda_{i}-\lambda_{j}\right)\left(2\left(\lambda_{i}+\lambda_{j}\right)-\sum_{k=1}^{3} \lambda_{k}\right)=0, \quad \text { for } \quad i \neq j
$$

From (3.2), we can easily show that the following cases are possible:

$$
\begin{aligned}
& \text { (i) } \lambda_{1}=\lambda_{2}=\lambda_{3}=\lambda \neq 0, \\
& \text { (ii) } \lambda_{1}=\lambda_{2}=\lambda \neq 0, \quad \lambda_{3}=0, \\
& \text { (iii) } \lambda=\lambda_{2}=\lambda_{3}=0 .
\end{aligned}
$$

Thus we have

PROPOSITION 3.1. Let $M$ be a 3-dimensional connected Riemannian manifold satisfying the condition (**). If the rank of $R$ is 3 at least at one point of $M$, then $M$ is a space of constant curvature.

REMARK. In general, if a Riemannian manifold $(M, g)$ satisfies the condition $(* *)$, then we can see that multiplicity of any non-zero eigenvalue of $A$ is greater than 1 .

Next, we assume that the rank of the Ricci form $R_{1}$ is 2 at some point $x_{0} \in M$. Then we can define two differentiable distributions, $T_{1}$ and $T_{0}$ corresponding to the eigenvalues, $\lambda$ and 0 of $A$ respectively on $W_{0}$, and furthermore we may choose a differentiable field of orthonormal basis $\left\{X_{1}, X_{2}, X_{3}\right\}$ near $x$ in $W_{0}$, for any $x \in W_{0}$, in such a way that $\left\{X_{1}, X_{2}\right\}$ and $\left\{X_{3}\right\}$ are bases for $T_{1}$ and $T_{0}$ respectively, where $W_{0}$ is the connected component of $x_{0}$ in $W=\{x \in M$; the rank of $R_{1}$ is 2 at $x$ \}.

With respect to the basis $\left\{X_{1}, X_{2}, X_{3}\right\}$, we have

$$
\begin{gathered}
R\left(X_{1}, X_{2}\right)=\lambda X_{1} \wedge X_{2}, \quad \text { and otherwise zero. } \\
R_{1}\left(X_{1}, X_{1}\right)=R_{1}\left(X_{2}, X_{2}\right)=\lambda, \quad \text { and otherwise zero. }
\end{gathered}
$$

Now, we assume that $M$ is conformally flat and complete. Then the following equation holds good : 


$$
\begin{aligned}
& \left(\nabla_{\boldsymbol{Z}} R_{1}\right)(X, Y)-\left(\nabla_{Y} R_{1}\right)(X, Z) \\
& \quad=(1 / 4)(Z(\text { trace } A) g(X, Y)-Y(\text { trace } A) g(X, Z)),
\end{aligned}
$$

for any tangent vectors $X, Y$ and $Z$ of $M$.

We shall prove

PROPOSITION 3.2. Let $M$ be a 3-dimensional connected complete conformally flat space satisfying the condition (**). If the rank of the Ricci form $R_{1}$ is 2 , then $M$ is a locally product space of a 2-dimensional space of constant curvature and 1-dimensional space.

PROOF. From (2.5) and (3.3), by the second Bianchi identity, we have

$$
\gamma_{331}=\gamma_{332}=0 \text {. }
$$

By putting $X=X_{1}, Y=X_{2}, Z=X_{3}$ in (3.5) and using (2.5) and (3.4), we have

$$
\gamma_{213}=0, \quad \text { similarly, } \quad \gamma_{123}=0 .
$$

By putting $X=X_{3}, Y=X_{1}, Z=X_{3}$

$$
X_{1} \lambda=0, \quad \text { similarly, } \quad X_{2} \lambda=0 .
$$

By putting $X=X_{1}, Y=X_{1}, Z=X_{3}$

$$
X_{3} \lambda+2 \lambda \gamma_{131}=0, \quad \text { similarly, } \quad X_{3} \lambda+2 \lambda \gamma_{232}=0 .
$$

By (3.9), we have

$$
\gamma_{31}=\gamma_{232} \text {. }
$$

From the equation $R\left(X_{1}, X_{3}\right) X_{3}=0$, by making use of (3.6), (3.7) and (3.10), we have

$$
X_{3} \gamma_{131}+\left(\gamma_{131}\right)^{2}=0
$$

Therefore, from the above discussions, the rest of proof is given by the slight modifications of the arguments in the last case in 2 . 


\section{REFERENCES}

[1] S. Kobayashi AND K. Nomizu, Foundations of Differential Geometry, Vol. I, Interscience Publishers, New York, 1963.

[2] M. KURITA, On the holonomy group of conformally flat Riemannian manifold, Nagoya Math. J., 9 (1955), 161-171.

[ 3 ] K. NomizU, On hypersurfaces satisfying a certain condition on the curvature tensor, Tôhoku Math. J., 20(1968), 46-59.

[4] M. TANI, On a conformally flat Riemannian space with positive Ricci curvature, Tohoku Math. J., 19 (1967), 227-231.

[5] S. TANNO, Hypersurfaces satisfing a certain condition on the Ricci tensor, Tohoku Math. J., 21(1969), 297-303.

FACULTY OF SCIENCE

NIIGATA UNIVERSITY

NIIGATA, JAPAN 
